\title{
EFFECTS OF DEXTRAN, POLYVINYLPYRROLIDONE AND GAMMA GLOBULIN ON THE HYPERLIPIDEMIA OF EXPERIMENTAL NEPHROSIS *
}

\author{
By JAMES C. ALLEN, $†$ JAMES H. BAXTER AND HOWARD C. GOODMAN \\ (From the Laboratory of Cellular Physiology and Metabolism, National Heart Institute, \\ Bethesda, $M d$.)
}

(Submitted for publication August 12, 1960 ; accepted November 3, 1960)

During the treatment of edema in nephrotic patients by the infusion of dextran, Mollison and Rennie (1) noted falls in serum cholesterol concentration which they believed were due to dilution but which persisted after hematocrits had returned to pretreatment levels, and which by calculation from their data were greater than could be accounted for by hemodilution alone. Subsequently Soothill and Kark (2) and Baxter, Goodman and Havel (3) demonstrated that the intravenous infusion of human albumin into nephrotic patients resulted in a significant lowering of serum lipid concentrations. In their studies on nephrotic rats Rosenman and Friedman (4) demonstrated that the infusion of bovine albumin into these animals lowered serum lipid concentrations. Heymann, Nash, Gilkey and Lewis (5) treated nephrotic rats with dextran and noted significant reductions in the serum concentrations of total lipid and cholesterol. They recognized that these reductions resulted from more than hemodilution, but offered no explanation for this effect. This study was undertaken to investigate more extensively the effects of the administration of dextran on the hyperlipidemia of experimental nephrosis in rats, and to see whether these effects were shared by osmotically active macromolecular substances other than dextran and albumin.

\section{METHODS}

Female rats of the Osborne-Mendel strain, weighing approximately $200 \mathrm{~g}$, were made nephrotic by the intravenous injection of an aliquot of pooled antirat-kidney rabbit serum prepared as previously reported from this laboratory (6). Eleven days after injection of the antiserum a control blood sample of approximately $1.2 \mathrm{ml}$ volume was obtained by heart puncture after a 6 hour

* A preliminary report of this work appeared as an abstract (Clin. Res. 1959, 7, 278).

$\dagger$ Present address: Department of Medicine, Johns Hopkins Hospital, Baltimore, Md. fast. On Days 12, 13 and 14 after injection of the antiserum the rats received, twice daily, intraperitoneal injections of dextran, polyvinylpyrrolidone (PVP), bovine $\gamma$-globulin, bovine albumin or salt in solutions as described below. On Day 15 following antiserum injection, approximately 20 hours after the last intraperitoneal injection and again after a 6 hour fast, the final blood sample was obtained by aspiration from the exposed abdominal aorta. Both heart puncture and aortic aspiration were done under ether anesthesia. Both blood samples were obtained through clean, dry needles into heparinized syringes. Normal control animals were handled as were the nephrotic rats except for the initial injection of antiserum. The animals were housed in metabolic cages with free access to water and Purina pellet diet, save for the presampling fasting periods when pellets were withheld. Twenty-four-hour urine collections were made from Days 11 through 15 after the injection of the antiserum and were used for the determination of urinary protein content.

Hematocrits were determined in duplicate on each blood sample by the capillary microhematocrit method immediately after each sample was obtained. Because of the observed ability of dextran and PVP to increase the erythrocyte sedimentation rate, careful mixing and rapid loading of the capillary tubes were insured. These hematocrits were used for calculation of plasma volume changes during the experimental period by the formula

fractional change in plasma volume

$$
\begin{aligned}
& =\frac{\text { plasma volume }_{2}-\text { plasma volume }_{1}}{\text { plasma volume }_{1}} \\
& =\frac{\left(\text { hematocrit }_{1} / \text { hematocrit }_{2}\right)-1}{1-\left(\text { hematocrit }_{1} / 100\right)}
\end{aligned}
$$

where hematocrit ${ }_{1}$ and hematocrit ${ }_{2}$ represent the average hematocrits obtained at the first and second blood samplings, respectively. Plasma concentrations of albumin and lipids obtained following treatment with the macromolecular substances have been corrected for such plasma volume changes. The calculation presented in the formula above is based on the assumption that the circulating red cell mass remains constant during the experimental period. A decrease in the red cell mass during treatment would lead to- overcorrection, thus minimizing the changes shown by these data. A few animals had evidence of intraperitoneal, gastrointestinal 
or external bleeding as a result of the injections; these were not included in the study.

Plasma was separated from the blood samples by centrifugation and stored at $5^{\circ} \mathrm{C}$ in stoppered containers for 1 to 4 days before lipid extraction and the various other determinations described below. Before aliquots were taken for analysis, the plasma samples were brought to $37^{\circ} \mathrm{C}$ and mixed carefully by inversion. Aliquots were extracted with chloroform-methanol by the method of Havel (7). Aliquots of the extracted lipids were used for the determination of total lipids by the method of Bragdon (8), total cholesterol by the method of Zak and co-workers (9), and phospholipids by a modification of the method of Stewart and Hendry (10), using a factor of 25 for the conversion of lipid phosphorus to phospholipid. Only those nephrotic rats with initial total lipid concentrations of $500 \mathrm{mg}$ per $100 \mathrm{ml}$ or greater (approximately twice the mean normal value in this laboratory) were used in the study. All of the methods were standardized for microdeterminations. Plasma albumin concentrations were determined by a modification of the dye interaction method of Rutstein, Ingenito and Reynolds (11). Determinations of dextran (12) and PVP (13) were performed on the appropriate samples. Total plasma protein concentrations and urinary protein content were determined by modifications of the method of Hiller, Greif and Beckman (14).

By the method of Bragdon (8) plasma triglyceride concentration may be determined by appropriate calculations from plasma total lipid, phospholipid, and total and free cholesterol concentrations. Because of the small volume of plasma available, free cholesterol determinations were not performed on the experimental animals. In separate groups of normal and nephrotic rats, however, free and total cholesterol concentrations were determined by a modification of the Schoenheimer-Sperry method (15). Free cholesterol to total cholesterol ratios of the nephrotic and normal groups were, respectively, 0.28 (SD 0.01) and 0.32 (SD 0.01). By use of these ratios the plasma concentration of free cholesterol was approximated in each animal from the total cholesterol value, and triglyceride concentrations were then calculated. It is recognized that the free cholesterol to total cholesterol ratio may have changed following treatment with these macromolecular substances, but a significant change in this ratio will introduce only a small error into the calculated triglyceride concentration.

Both extraction procedures and chemical determinations were checked for reliability in the presence of the various macromolecular substances used in this study by in vitro addition and duplicate determination. No interference was demonstrated except in the determination of total lipids by the dichromate-reduction method of Bragdon in the presence of PVP. Various extraction methods were tried in an effort to remove PVP from the lipid phase, all of which were unsuccessful owing to the solubility of that compound in both polar and nonpolar solvents. Hence, for the animals treated with PVP, total lipid concentrations were derived from determinations of plasma carboxylic acid ester content done by a modification of the method of Rapport and Alonzo (16). In a separate group of nephrotic rats, total lipid determinations by the two methods showed the dichromate reduction method to give values approximately 1.5 times those determined by the ester method. This ratio cannot, of course, be justified for values obtained after PVP treatment. Triglyceride concentrations for PVPtreated rats are not presented, since they cannot be compared with those calculated from dichromate-reduction total lipid determination.

Dextran was administered as a commercially supplied 6 per cent solution in isotonic saline (Gentran, Baxter Laboratories, Inc.) in amounts of 0.3 to $1.4 \mathrm{~g}$ per day. The dextran in this preparation has an average molecular weight of 75,000. Commercially prepared powdered PVP (fraction K-30 from General Aniline and Film Corp.) was fractionated by the isopropanol-acetone method outlined by Levy, Caldas and Fergus (17), to obtain a sample with an average molecular weight of 75,000 as determined by viscosity measurement. This was dissolved to a 6 per cent solution in isotonic saline and injected intraperitoneally in amounts of 0.2 to $1.0 \mathrm{~g}$ per day. Bovine albumin prepared by Armour Pharmaceutical as bovine plasma fraction $\mathrm{V}$ was administered as 10 to 20 per cent solutions in isotonic saline in amounts of 1.2 to $4.0 \mathrm{~g}$ per day. Bovine $\gamma$-globulin (Armour) prepared as bovine plasma fraction II was administered as 15 to 20 per cent solutions in isotonic saline in amounts of 2.0 to $4.0 \mathrm{~g}$ per day. A group of nephrotic rats received twice daily intraperitoneal injections of isotonic saline solution in a volume equal to the largest volume of injection received by any of the experimental animals $(9 \mathrm{ml})$. In general, normal control rats received smaller amounts of the various agents than their nephrotic counterparts, but achieved comparable plasma concentrations owing to the smaller urinary loss.

Lipoprotein lipase activity was assayed in both normal and nephrotic rats following the intravenous injection of $1 \mathrm{mg}$ of heparin, 3 to $150 \mathrm{mg}$ of dextran, 90 $\mathrm{mg}$ of PVP, and $195 \mathrm{mg}$ of $\gamma$-globulin. All injections were made in a standard volume of $1 \mathrm{ml}$ and the amounts administered were calculated to provide plasma concentrations comparable with those determined following the intraperitoneal treatments outlined above. Fifteen minutes after the intravenous injections the rats were bled into heparinized syringes from the abdominal aorta under ether anesthesia. The plasma was separated immediately by centrifugation, and $1-\mathrm{ml}$ aliquots were added to cuvets containing a commercial coconut oil emulsion (Ediol, Schenley Laboratories) diluted 1 to 50 with normal saline. The cuvets were held in a water bath at $37^{\circ} \mathrm{C}$ and optical densities were read in a Coleman Jr. spectrophotometer at $660 \mathrm{~m} \mu$ immediately and at 5-minute intervals for up to 140 minutes. Clearing was followed in duplicate experiments with and without the in vitro addition of 25 to $30 \mathrm{mg}$ of albumin in the form of a 15 per cent solution of Armour fraction $\mathrm{V}$ bovine albumin. Clearing activity was also assayed in postheparin plasma following the in vitro addition of $30 \mathrm{mg}$ of dextran or PVP. Plasma obtained from rats follow- 
DEXTRAN, PVP, GAMMA GLOBULIN AND NEPHROTIC LIPEMIA

TABLE I

Mean plasma concentrations attained by the intraperitoneal administration of dextran, polyvinylpyrrolidone $(P V P), \gamma-$ globulin and albumin to nephrotic and normal rats

\begin{tabular}{|c|c|c|c|c|c|}
\hline \multirow{3}{*}{$\begin{array}{c}\text { Substance } \\
\text { injected }\end{array}$} & \multirow{3}{*}{$\begin{array}{l}\text { Substance } \\
\text { determined }\end{array}$} & \multicolumn{4}{|c|}{ Concentrations } \\
\hline & & \multicolumn{2}{|c|}{ Nephrotic rats* } & \multicolumn{2}{|c|}{ Normal rats* } \\
\hline & & Before & After & Before & After \\
\hline & & \multicolumn{4}{|c|}{$\mathrm{g} / 100 \mathrm{ml}$ plasma } \\
\hline Dextran & Dextran & 0 & $1.6 \pm 0.2 \dagger$ & 0 & $1.6 \pm 0.1$ \\
\hline PVP & PVP & 0 & $\begin{array}{l}(0.3-3.0) f \\
1.7 \pm 0.1 \\
(1.2-2.5)\end{array}$ & 0 & $\begin{array}{l}(1.5-2.1 \\
1.4 \pm 0.1 \\
(1.1 \pm 1.7)\end{array}$ \\
\hline$\gamma$-Globulin & $\begin{array}{l}\text { Total } \\
\text { protein }\end{array}$ & $\begin{array}{c}4.9 \pm 0.2 \\
(3.7-6.2)\end{array}$ & $\begin{array}{c}7.3 \pm 0.2 \\
(6.2 \pm 8.9)\end{array}$ & $\begin{array}{c}6.8 \pm 0.2 \\
(6.5-7.0)\end{array}$ & $\begin{array}{c}9.7 \pm 0.3 \\
(8.5=10.2)\end{array}$ \\
\hline Albumin & Albumin & $\begin{array}{l}1.8 \pm 0.2 \\
(1.1-3.4)\end{array}$ & $\begin{array}{c}2.5 \pm 0.2 \\
(1.4-3.9)\end{array}$ & & \\
\hline
\end{tabular}

* All $\mathrm{p}$ values for significance of the difference between before and after concentrations are $<0.01$.

† Standard error of the mean.

$\ddagger$ Range of values.

ing 3 days of twice daily intraperitoneal injections of dextran or PVP was also checked for clearing activity. All readings were corrected to a control optical density of 0.276 .

Statistical analysis of the data to be presented was performed by the methods outlined by Stearman (18).

\section{RESULTS}

Animals. In general, both nephrotic and normal rats tolerated the injections and the macromolecular substances well. The reported idiosyncrasy of rats to dextran (19) was not observed during these experiments. Most of the animals showed no gross change in food intake and appeared active and healthy throughout the experiment. A number (representing less than one-fourth of the experimental animals) which received the largest volumes by injection appeared lethargic and had roughened fur and diminished food intake. Body weights increased slightly over the 3-day injection period. At the time of the final bleeding the abdominal cavities were inspected. In no animal was there evidence of intraperitoneal infection, and the peritoneal surfaces remained smooth and glistening. As mentioned, a few animals had evidence of intraperitoneal, retroperitoneal or gastrointestinal bleeding consequent to trauma at the time of an injection; such animals were not considered in the following data. Except for the small percentage of animals that received the largest volumes by intraperitoneal injection, absorption of the administered solutions was grossly complete at the time of the final bleeding. Average 24-hour urine volumes of the various groups increased 1.1 to 1.5 times the control volume.

Effects of the administered substances. As can be seen from Table I the intraperitoneal administration of dextran and PVP to nephrotic rats resulted in mean plasma concentrations of these macromolecules of between 1 and $2 \mathrm{~g}$ per cent. It is true, of course, that rapid clearance of the smaller molecules by the nephrotic kidneys resulted in a circulating macromolecular population with a higher average molecular weight than that administered. In those animals receiving $\gamma$-globulin there were significant increases in total plasma

TABLE II

Mean hematocrits before and after treatment of nephrotic and normal rats with dextran, $P V P, \gamma-$ globulin, albumin or saline

\begin{tabular}{lccc}
\hline \multirow{2}{*}{ Treatment } & \multicolumn{3}{c}{ Hematocrits } \\
\cline { 2 - 4 } & Before & After & $\mathrm{p}^{*}$ \\
\hline Nephrotic rats & \multicolumn{3}{c}{ \% by volume } \\
$\quad$ Dextran & $39.7 \pm 0.7 \dagger$ & $34.5 \pm 1.2$ & $<0.01$ \\
PVP & $40.3 \pm 0.8$ & $30.8 \pm 1.0$ & $<0.01$ \\
$\gamma$-Globulin & $40.7 \pm 1.0$ & $31.2 \pm 0.7$ & $<0.01$ \\
Albumin & $40.3 \pm 1.0$ & $38.6 \pm 2.0$ & $>0.05$ \\
Saline & $40.1 \pm 2.0$ & $39.8 \pm 2.0$ & $>0.05$ \\
Normal rats & & & \\
Dextran & $50.3 \pm 1.0$ & $39.0 \pm 0.5$ & $<0.01$ \\
PVP & $51.6 \pm 2.4$ & $36.9 \pm 1.1$ & $<0.01$ \\
$\gamma$-Globulin & $52.7 \pm 1.5$ & $32.8 \pm 0.8$ & $<0.01$ \\
& & & \\
& &
\end{tabular}

* Significance of the difference between before and after values.

t Standard error of the mean. 


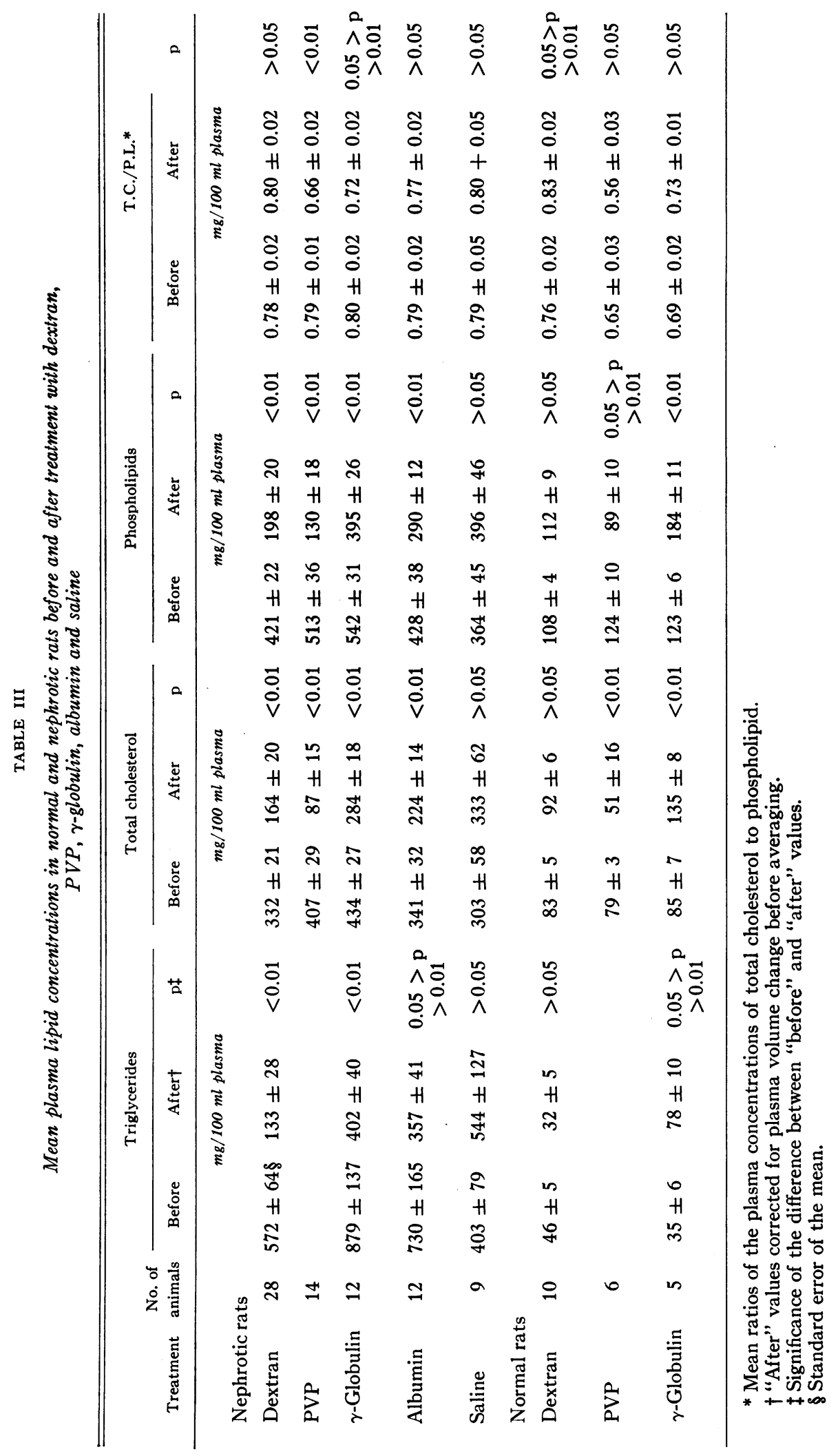


protein concentration, the increment presumably contributed by the injected globulin. The small mean increase in plasma albumin concentration noted here is undoubtedly a function of the rapid clearance of circulating albumin in these nephrotic animals. Plasma concentrations of these substances following their injection into normal rats were of comparable mean value, although narrower in range.

That the plasma concentrations attained were effective in increasing plasma volume, as indicated by a fall in hematocrit over the treatment period, is shown in Table II. There were statistically significant falls in hematocrit in all groups except the nephrotic rats which received albumin or isotonic saline. No significant change in hematocrit was observed unless the plasma albumin concentration increased by $1 \mathrm{~g}$ per cent or more, or the plasma dextran concentration was $1 \mathrm{~g}$ per cent or greater. Injection of $\gamma$-globulin and PVP caused significant plasma volume expansion in all of the animals presented in this data. Although it was not determined in this study, plasma concentrations of the various macromolecular substances were undoubtedly higher in the hours immediately after each injection than those represented in Table I. One would expect that even in those animals with the lowest final plasma concentrations of dextran and albumin there was some degree of plasma volume expansion following each injection.

The data in Table III demonstrate that the administration of dextran, PVP, $\gamma$-globulin and albumin to nephrotic rats resulted in significant decreases in the plasma concentrations of triglyceride, total cholesterol, and phospholipids. The injection of isotonic saline solution intraperitoneally into nephrotic rats caused no significant changes in the plasma concentrations of these lipid fractions. When administered to normal rats, dextran caused no significant changes in the concentration of triglyceride, total cholesterol or phospholipids, while PVP significantly lowered total cholesterol and phospholipid concentrations. The administration of $\gamma$-globulin to normal rats apparently caused an increase in the concentrations of these lipid fractions. Although this increase may have been due to increased lipidemia resulting from the $\gamma$-globulin treatment, it probably represents overcorrection of the final
TABLE IV

\begin{tabular}{|c|c|c|c|}
\hline \multirow[b]{2}{*}{ Treatment } & \multicolumn{3}{|c|}{ Fractional decrease* } \\
\hline & Triglycerides & Total cholesterol & Phospholipids \\
\hline \multicolumn{4}{|c|}{ Nephrotic rats } \\
\hline Dextran & $0.76 \pm 0.04 \dagger$ & $0.52 \pm 0.04$ & $0.53 \pm 0.04$ \\
\hline PVP & & $0.79 \pm 0.03$ & $0.74 \pm 0.03$ \\
\hline$\gamma$-Globulin & $0.49 \pm 0.08$ & $0.33 \pm 0.04$ & $0.25 \pm 0.05$ \\
\hline Albumin & $0.31 \pm 0.12$ & $0.30 \pm 0.07$ & $0.28 \pm 0.06$ \\
\hline \multicolumn{4}{|l|}{ Normal Rats } \\
\hline PVP & & $0.36 \pm 0.09$ & $0.27 \pm 0.09$ \\
\hline
\end{tabular}

* Calculated as the ratio of the change in concentration over the experimental period to the control concentration. Post-treatment concentrations were corrected for plasma volume change.

$\dagger$ Standard error of the mean.

plasma lipid concentrations for plasma volume change. The intraperitoneal administration of $6 \mathrm{mg}$ of dextran per day to three nephrotic animals resulted in no change in their hematocrit or plasma lipid concentrations, and no circulating dextran could be demonstrated at the time of the final bleeding.

Table IV summarizes the fractional changes in the various lipid moieties calculated as the ratio of their change in concentration to their original concentration. It can be seen that following injection of dextran and $\gamma$-globulin most marked changes had occured in the plasma concentration of triglyceride. This selective effect on plasma triglyceride was also demonstrated following administration of albumin to those nephrotic rats with the highest triglyceride concentration. This finding is not apparent from the mean values in Table IV owing to the wide variation in control plasma triglyceride concentration in the albumintreated group, and the variable response of those animals with only slightly elevated triglyceride levels. Table IV also demonstrates that decreases in plasma total cholesterol and phospholipid concentration during administration of PVP were proportionally greater in nephrotic than in normal animals. When carboxylic acid ester total lipid concentration was converted to dichromate total lipid concentration (see Methods), the subsequent calculation of triglyceride concentration in a number of animals indicated that PVP, as well 
TABLE V

Mean proteinuria and plasma albumin concentrations in nephrotic and normal rats treated with dextran, PVP, $\gamma$-globulin, albumin or saline

\begin{tabular}{|c|c|c|c|c|c|c|}
\hline \multirow[b]{2}{*}{ Treatment } & \multicolumn{3}{|c|}{ Proteinuria } & \multicolumn{3}{|c|}{ Plasma albumin conc. } \\
\hline & Control & During & $\mathrm{p}^{*}$ & Before & Aftert & $\mathrm{p}^{*}$ \\
\hline & \multicolumn{3}{|c|}{$m g$ protein $/ h r$} & \multicolumn{3}{|c|}{$\mathrm{g} / 100 \mathrm{ml}$ plasma } \\
\hline Nephrotic rats & & & & & & \\
\hline Dextran & $14.0 \pm 1.2$ & $12.2 \pm 1.2$ & $\begin{array}{c}0.05>0 \\
>0.01\end{array}$ & $2.1 \pm 0.1$ & $2.3 \pm 0.1$ & $>0.05$ \\
\hline PVP & $16.4 \pm 1.5$ & $14.6 \pm 1.2$ & $>0.05$ & $1.7 \pm 0.1$ & $2.0 \pm 0.1$ & $<0.01$ \\
\hline$\gamma$-Globulin & $18.4 \pm 2.3$ & $35.0 \pm 3.5$ & $<0.01$ & $1.6 \pm 0.2$ & $1.9 \pm 0.1$ & $\underset{>0.01}{0.05}>p$ \\
\hline Albumin & $16.9 \pm 2.1$ & $51.1 \pm 4.1$ & $<0.01$ & $1.8 \pm 0.2$ & $2.5 \pm 0.2$ & $<0.01$ \\
\hline Saline & $13.6 \pm 1.8$ & $16.6 \pm 1.6$ & $\begin{array}{c}0.05>p \\
<0.01\end{array}$ & $1.8 \pm 0.2$ & $1.9 \pm 0.2$ & $>0.05$ \\
\hline \multicolumn{7}{|l|}{ Normal rats } \\
\hline Dextran & $0.1 \pm 0.0$ & $0.2 \pm 0.0$ & $>0.05$ & $4.9 \pm 0.1$ & $5.5 \pm 0.2$ & $\underset{>0.01}{0.05}>\mathrm{p}$ \\
\hline PVP & $0.2 \pm 0.0$ & $0.2 \pm 0.0$ & $>0.05$ & $4.9 \pm 0.4$ & $5.5 \pm 0.8$ & $>0.05$ \\
\hline$\gamma$-Globulin & $0.2 \pm 0.0$ & $0.2 \pm 0.0$ & $>0.05$ & $5.4 \pm 0.1$ & $6.2 \pm 0.3$ & $>0.05$ \\
\hline
\end{tabular}

* Significance of the difference between before and after or during values.

$\dagger$ "After" values corrected for plasma volume change before averaging.

as the other macromolecular substances, lowered plasma triglyceride more than plasma total cholesterol or phospholipid. This calculation in many of the animals, however, yielded impossible (i.e., negative) values, indicating the invalidity in this method of applying the same ratio for conversion of lipid concentrations both before and after PVP treatment. The ratios of total cholesterol to phospholipid concentration (Table III) showed in general only moderate and varying changes following administration of these substances. After treatment of nephrotic rats with

TABLE VI

Mean fractional decreases * in plasma lipid concentrations of nephrotic rats with high and low plasma dextran levels

\begin{tabular}{lccc}
\hline \hline & $\begin{array}{c}\text { Dextran conc. } \\
>2 \mathrm{~g} \%\end{array}$ & $\begin{array}{c}\text { Dextran conc. } \\
<1 \mathrm{~g} \%\end{array}$ & $\mathrm{p} \dagger$ \\
\hline $\begin{array}{l}\text { Number of rats } \\
\begin{array}{l}\text { Triglycerides } \\
\text { Total } \\
\text { cholesterol }\end{array}\end{array}$ & $0.95 \pm 0.01 \ddagger$ & $0.54 \pm 0.01$ & $<0.01$ \\
$\begin{array}{c}\text { Phospholipids } \\
\text { hho. }\end{array}$ & $0.71 \pm 0.02$ & $0.36 \pm 0.01$ & $<0.01$ \\
\hline
\end{tabular}

* Calculated as the ratio of the change in concentration to the control concentration. Post-treatment concentrations were corrected for plasma volume change.

+ Significance of the difference between the means of the two groups.

\pm Standard error of the mean.
PVP, however, there was a significant fall in this ratio.

From Table $\mathrm{V}$ it is evident that nephrotic animals that received dextran, PVP and $\gamma$-globulin had a slight increase in their mean corrected plasma albumin concentration during the experimental period, which in no instance was greater than $0.3 \mathrm{~g}$ per cent and which was not statistically significant in the dextran group. This slight rise was coupled with a decrease of less than 2 mg per hour in the mean proteinuria of those nephrotic rats receiving dextran and PVP. It should be noted that this increase in serum albumin concentration and decrease in proteinuria represent mean figures, and a number of rats (11 of 28 treated with dextran, 5 of 14 treated with PVP, and 4 of 12 treated with $\gamma$-globulin) showed no change, or the opposite change, in plasma albumin concentration concomitant with decreases in their hyperlipidemia. The administration of $\gamma$-globulin to nephrotic rats caused marked increases in their proteinuria, but essentially none of the $\gamma$-globulin administered to normal rats appeared in their urine during the treatment period. Though not statistically significant in these groups, it is of interest that the mean posttreatment albumin concentrations of all three groups of normal animals were higher than their 
mean control values. This may represent overcorrection for plasma volume change.

In general, the higher the plasma concentration of the various macromolecular substances, the greater was the decrease in plasma lipid concentration. This relationship was noted following administration of dextran, PVP, $\gamma$-globulin and albumin, although there was considerable variation in the magnitude of the response of different animals to comparable plasma concentrations of these macromolecules. Table VI establishes the statistical validity of this observation for dextrantreated nephrotic rats of high and low plasma dextran concentrations.

Lipoprotein lipase assay. Figure 1 presents typical experiments in which plasma lipoprotein lipase activity is determined by its effect on clearing of a coconut oil suspension. By this method lipolytic activity is demonstrable in the plasma of both normal and nephrotic rats following intravenous administration of heparin. Note that when plasma obtained following the administration of heparin to normal rats is incubated with the coconut oil suspension, the initial phase of clearing is followed by a phase of increasing turbidity, as previously described by French, Robinson and Florey (20). Fortification of the clearing system with albumin enhances clearing and inhibits the return of turbidity, presumably owing to binding of the liberated fatty acids by albumin (21).

As is shown in Figure 1 we were unable to demonstrate that dextran, PVP, or $\gamma$-globulin, when administered intravenously in a variety of doses, shared heparin's ability to release lipoprotein lipase. Plasma obtained following a 3-day course of intraperitoneal injections of dextran or PVP showed no lipolytic activity. There was similarly no evidence that dextran, PVP or $\gamma$-globulin shared albumin's ability to enhance clearing by binding free fatty acids.

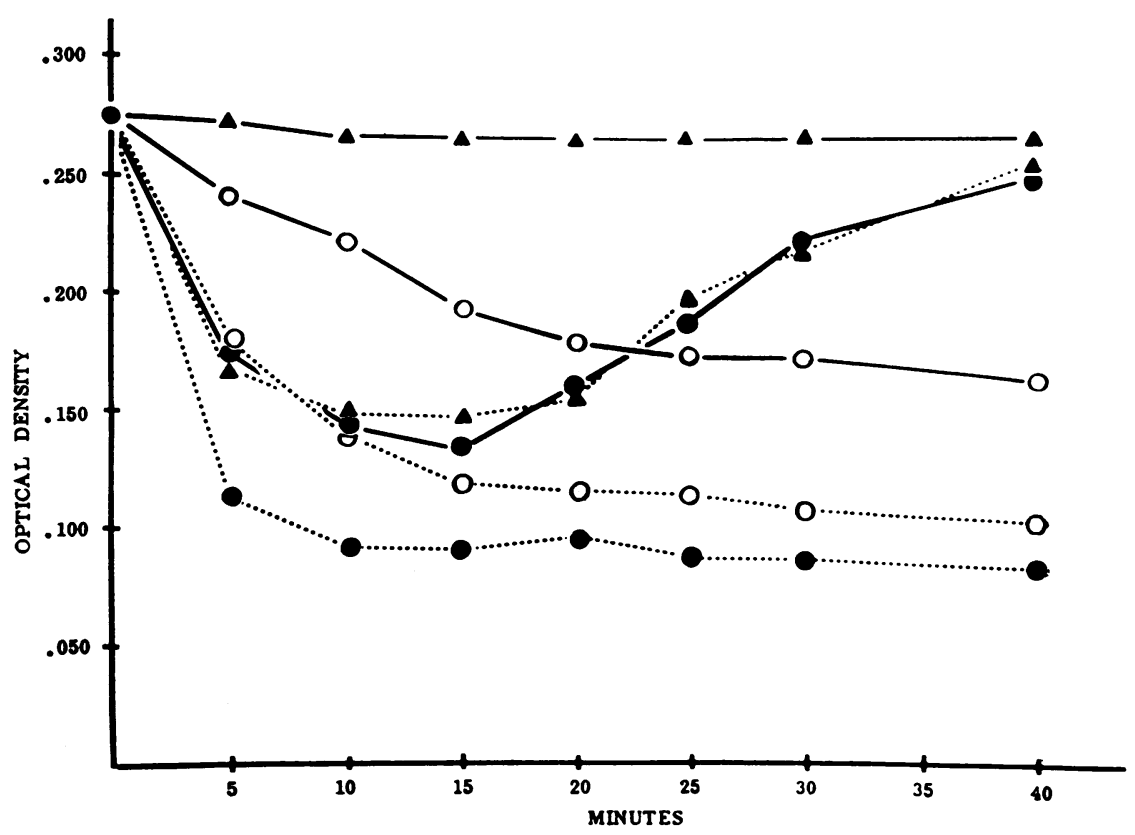

Fig. 1. Clearing of coconut oil Suspension by Rat plasma (Representative EXPERIMENTS). Plasma obtained from: - Normal rat after intravenous heparin (see text for dosage). $\bullet \cdots \cdot$ Normal rat after intravenous heparin; clearing system fortified with albumin. $\mathbf{\Delta} \cdots \boldsymbol{\Delta}$ Normal rat after intravenous heparin; clearing system fortified with dextran, polyvinlypyrrolidone (PVP) or bovine $\gamma$-globulin. $\bigcirc-O$ Nephrotic rat after intravenous heparin (fortification of clearing system with dextran, PVP or bovine $\gamma$-globulin yielded identical results). $\bigcirc \cdots \bigcirc$ Nephrotic rat after intravenous heparin; clearing system fortified with albumin. $\Delta-\boldsymbol{\Delta}$ Normal or nephrotic rat after intravenous dextran, PVP or bovine $\gamma$-globulin. 


\section{DISCUSSION}

From the foregoing data it is evident that administration of dextran, PVP, bovine $\gamma$-globulin and bovine albumin can cause significant reductions in the hyperlipidemia of nephrotic rats. Though PVP apparently has some differential effect on the total cholesterol and phospholipid fractions, the general pattern of the plasma lipids following administration of these various substances to nephrotic rats is similar, making the hypothesis of a common mechanism for their action an appealing one.

It is impossible to delineate conclusively a mechanism for this lipid-lowering effect from the data presented in this report, but a number of possible mechanisms can be reasonably excluded on the basis of information available here. As judged by plasma albumin concentrations and proteinuria, there is no evidence of any change in the nephrotic status of these animals during the period of study. Similarly, the general appearance and behavior of the rats during the study did not suggest any general toxic effect of the administered substances, nor was there overt evidence of systemic effects from these substances in any but a small percentage of the animals. This observation is in agreement with those of Heymann and colleagues (5) who noted prompt re-elevation of serum lipids in their nephrotic rats following cessation of dextran therapy, and who demonstrated no azotemia following the use of this substance. A pilot experiment on the in vitro synthesis of lipoproteins by liver slices taken from nephrotic rats which had received dextran or PVP has been performed by the method of Radding, Bragdon and Steinberg (22). There was no gross impairment of hepatic lipoprotein synthesis in these rats compared with untreated nephrotic controls, although minor differences cannot be excluded from the data obtained (23).

In the experiments presented here we could find no evidence that acute or chronic administration of dextran, PVP or $\gamma$-globulin resulted in the release of lipoprotein lipase in the intact normal or nephrotic rat. This is of interest in that a number of anionic polysaccharides and polyelectrolytes, including heparin and dextran sulfate, do have this capability (24-27), and there is little doubt from the work of Opdyke and coworkers (28), Basu and Stewart (29), and
Cohen and Tudhope (30) that chronic administration of substances which release lipoprotein lipase will significantly affect all of the fractions of blood lipids. Our inability to demonstrate lipoprotein lipase release by the compounds used in this study is in agreement with the observations of Stewart (31) who could demonstrate no effect of dextran comparable with that of heparin and dextran sulfate on increasing the electrophoretic mobility of lipoproteins, an effect ascribed by Gordon (32) to lipoprotein binding of the unesterified fatty acids released during lipolysis. As Gordon and co-workers (21) have shown, albumin potentiates lipoprotein lipase clearance by acting as a receptor for unesterified fatty acids. Under conditions in which we could demonstrate that albumin enhanced the lipoprotein lipase clearance reaction, we could demonstrate no comparable activity on the part of either dextran or PVP.

There are two possible mechanisms on which the present data give no information, but which would seem unlikely in the light of existing data. Both dextran and PVP are rapidly phagocytized by the reticuloendothelial system where they are stored for relatively long periods of time (33-35). The majority of existing evidence, however, indicates that serum cholesterol levels are raised rather than lowered by "blockade" of the reticuloendothelial system in rats and other animals (3638). That metabolism of dextran or PVP could affect the plasma lipids in these animals seems unlikely. PVP is apparently not metabolized (33) and dextran is only slowly metabolized (39) by animals. Serum lipid changes following carbohydrate feeding in rats differ from those demonstrated here (40), making metabolism of glucose units derived from dextran an unappealing mechanism of action.

Some of those animals receiving the largest volumes by injection had diminished food intake. It is impossible from our data to exclude subtle dietary changes in the remaining animals, or to exclude covert systemic reactions to the administered substances. Preliminary data by one of the authors (41), however, seem to indicate that even after prolonged periods ( 7 to 10 days) of limited food intake, or a day of absolute fasting, reductions in the plasma lipids of nephrotic rats are significantly less than those shown in these data. 
Although dextran, PVP, $\gamma$-globulin and albumin are of varying chemical structures, they share the ability to exert osmotic pressure in membrane systems. It is appealing to conclude, if only by exclusion, that this trait may cause at least a part of their lipid-reducing effects in nephrotic rats. The observations that similar patterns of lipid reduction result from the administration of these various substances, and that the magnitude of this reduction is in general directly proportional to the plasma concentration of the administered substance, are consistent with such a hypothesis. There are, of course, other conceivable mechanisms than those mentioned here, and as Baxter, Goodman and Havel (42) have pointed out, multiple factors undoubtedly influence nephrotic hyperlipidemia. Final elucidation of these various factors, as well as assessment of their relative importance, awaits further investigation.

\section{SUM MARY}

1. The administration of dextran, polyvinylpyrrolidone, bovine $\gamma$-globulin, and bovine albumin to nephrotic rats in doses sufficient to increase their plasma volume caused significant reductions in their plasma lipid concentrations.

2. Plasma triglyceride concentration decreased more than total cholesterol or phospholipid concentration during treatment with these substances.

3. Administration of these macromolecules to normal rats caused no significant fall in plasma lipid concentration, except in the case of polyvinylpyrrolidone which caused a decrease in total cholesterol and phospholipids. These reductions were proportionally less in normal rats than in nephrotic rats receiving polyvinylpyrrolidone.

4. In general, the magnitude of reduction of the plasma lipid concentrations was directly related to the plasma concentration of the macromolecular substance.

5. There was no significant change in the nephrotic state of the rats, as judged by plasma albumin concentration or proteinuria, adequate to explain the lipid changes.

6 . There was no demonstrable evidence that any of these compounds released lipoprotein lipase, or enhanced clearing of plasma lipids by acting as acceptors for free fatty acids.
7. Some mechanisms for this lipid-lowering effect are discussed. The possibility that the osmotic activity of these macromolecular substances is causally related to the falls in lipid concentration is mentioned.

\section{REFERENCES}

1. Mollison, A. W., and Rennie, J. B. Treatment of renal oedema with dextran. Brit. med. J. 1954, i, 893.

2. Soothill, J. A., and Kark, R. M. The effects of infusion of salt-poor human serum albumin on serum cholesterol cholinesterase, and albumin levels in healthy subjects and in patients ill with the nephrotic syndrome. Clin. Res. Proc. 1956, 4, 140.

3. Baxter, J. H., Goodman, H. C., and Havel, R. J. Hyperlipidemia of nephrosis (abstract). J. clin. Invest. 1957, 36, 873.

4. Rosenman, R. H., and Friedman, M. In vivo studies of the role of albumin in endogenous and heparin-activated lipemia-clearing in nephrotic rats. J. clin. Invest. 1957, 36, 700.

5. Heymann, W., Nash, G., Gilkey, C., and Lewis, M. Studies on the causal role of hypoalbuminemia in experimental nephrotic hyperlipemia. J. clin. Invest. $1958,37,808$.

6. Baxter, J. H., and Goodman, H. C. Nephrotoxic serum nephritis in rats. I. Distribution and specificity of the antigen responsible for the production of nephrotoxic antibodies. J. exp. Med. 1956, 104, 467.

7. Havel, R. J. Early effects of fat ingestion on lipids and lipoproteins of serum in man. J. clin. Invest. 1957, 36, 848.

8. Bragdon, J. H. Colorimetric determination of blood lipides. J. biol. Chem. 1951, 190, 513.

9. Zak, B., Dickenman, R. C., White, E. G., Burnett, H., and Cherney, P. J. Rapid estimation of free and total cholesterol. Amer. J. clin. Path. 1954, 24, 1307.

10. Stewart, C. P., and Hendry, E. B. The phospholipins of blood. Biochem. J. 1935, 29, 1683.

11. Rutstein, D. D., Ingenito, E. F., and Reynolds, W. E. The determination of albumin in human blood plasma and serum. A method based on the interaction of albumin with an anionic dye-2-(4'hydroxy-benzeneazo) benzoic acid. J. clin. Invest. 1954, 33, 211.

12. Semple, R. E. An accurate method for the estimation of low concentrations of dextran in plasma. Canad. J. Biochem. 1957, 35, 383.

13. Levy, G. B., and Fergus, D. Microdetermination of polyvinylpyrrolidone in aqueous solution and in body fluids. Analyt. Chem. 1953, 25, 1408.

14. Hiller, A., Greif, R. L., and Beckman, W. W. Determination of protein in urine by the biuret method. J. biol. Chem. 1948, 176, 1421. 
15. Sperry, W. M., and Webb, M. A revision of the Schoenheimer-Sperry method for cholesterol determination. J. biol. Chem. 1950, 187, 97.

16. Rapport, M. M., and Alonzo, N. Photometric determination of fatty acid ester groups in phospholipides. J. biol. Chem. 1955, 217, 193.

17. Levy, G. B., Caldas, I., Jr., and Fergus, D. Evaluation of polyvinylpyrrolidone preparations. Analyt. Chem. 1952, 24, 1799.

18. Stearman, R. L. Statistical concepts in microbiology. Bact. Rev. 1955, 19, 160.

19. Voorhees, A. B., Baker, H. J., and Pulaski, E. J. Reactions of albino rats to injections of dextran. Proc. Soc. exp. Biol. (N. Y.) 1951, 76, 254.

20. French, J. E., Robinson, D. S., and Florey, H. W. The effect of the intravenous injection of heparin on the interaction of chyle and plasma in the rat. Quart. J. exp. Physiol. 1953, 38, 101.

21. Gordon, R. S., Jr., Boyle, E., Brown, R. K., Cherkes, A., and Anfinsen, C. B. Role of serum albumin in lipemia clearing reaction. Proc. Soc. exp. Biol. (N. Y.) 1953, 84, 168.

22. Radding, C. S., Bragdon, J. H., and Steinberg, D. Synthesis of low- and high-density lipoproteins by rat liver in vitro. Biochim. biophys. Acta 1958, $30,443$.

23. Allen, J. C. Unpublished data.

24. Havel, R. J., and Bragdon, J. H. Heparin-like activity of polymetaphosphate. Circulation 1954, 10, 591.

25. Jeavons, S. M., and Ricketts, C. R. The effect of dextran sulphate injections on serum lipoproteins. J. clin. Path. 1956, 9, 255.

26. Glazko, A. J., Strong, J., and Kazenko, A. Some factors influencing the lipemia-clearing activity of rat plasma. Fed. Proc. 1954, 13, 55.

27. Houck, J. C., Morris, R. K., and Lazaro, E. J. Anticoagulant, lipemia clearing and other effects of anionic polysaccharides extracted from seaweed. Proc. Soc. exp. Biol. (N. Y.) 1957, 96, 528.

28. Opdyke, D. F., Rosenberg, A., Silber, R., Ott, W. H., and Siegel, H. Effect of chronic injection of a heparin complex on aortic atherosclerosis in cholesterol-fed chickens. J. Lab. clin. Med. 1955, 45, 270.

29. Basu, D. P., and Stewart, C. P. The effect of heparin on the plasma cholesterol. Edinb. med. J. 1950, 57, 596. •
30. Cohen, H., and Tudhope, G. R. Dextran sulfate: Use as an anticoagulant, and action in lowering serum cholesterol. Brit. med. J. 1956, 2, 1023.

31. Stewart, G. T. The action of sulphated polysaccharides upon serum lipids under clinical and experimental conditions. Brit. J. exp. Path. 1958, 39, 109.

32. Gordon, R. S., Jr. Interaction between oleate and the lipoproteins of human serum. J. clin. Invest. 1955, 34, 477.

33. Steele, R., Van Slyke, D. D., and Plazin, J. The fate of intravenously administered polyvinylpyrrolidone. Ann. N. Y. Acad. Sci. 1952, 55, 479.

34. Nelson, A. A., and Lusky, L. M. Pathological changes in rabbits from repeated intravenous injections of periston (polyvinyl pyrrolidone) or dextran. Proc. Soc. exp. Biol. (N. Y.) 1951, 76, 765.

35. Squire, J. R., Bull, J. P., Maycock, W. d'A., and Ricketts, C. R. Dextran: Its Properties and Use in Medicine. Springfield, Ill., Charles C Thomas, 1955 , pp. 26-27, 46-51.

36. Takagi, K. Alterations in cholesterol metabolism in rabbits after intravenous cholesterol injection: IV. Changes in blood cholesterol in rabbits with blocked reticulo-endothelial systems. Nagoya Igakkai Zasshi, 1936, 43, 597. (Chem. Abstr. 1937, 31, 7099.)

37. Brown, H. Z., Philips, I., and Kagan, B. M. The role of the reticuloendothelial system in vitamin A and cholesterol metabolism. Metabolism 1952, 1, 349.

38. Friedman, M., Byers, S. O., and Rosenman, R. H. Observations concerning the production and excretion of cholesterol in mammals. XII. Demonstration of the essential role of the hepatic reticuloendothelial cell (Kupffer cell) in the normal disposition of exogenously derived cholesterol. Amer. J. Physiol. 1954, 177, 77.

39. Cargill, W. H., and Bruner, H. D. Metabolism of C-14 labeled dextran in the mouse (abstract). J. Pharmacol. 1951, 103, 339.

40. Bragdon, J. H., Havel, R. J., and Gordon, R. S., Jr. Effects of carbohydrate feeding on serum lipids and lipoproteins in the rat. Amer. J. Physiol. 1957, 189, 63.

41. Baxter, J. H. Unpublished data.

42. Baxter, J. H., Goodman, H. C., and Havel, R. J. Serum lipid and lipoprotein alterations in nephrosis. J. clin. Invest. 1960, 39, 455. 